\title{
Business as usual? Welche Veränderungen setzen sich auch nach der Krise im Arbeitsalltag durch?
}

Die Schutzmaßnahmen rund um die COVID-19-Pandemie haben für zahlreiche Geschäftsbereiche Entwicklungen und Veränderungen angestoßen, die langfristig einen Wandel in unserer Arbeitswelt bewirken. Es ging dabei nicht nur darum, wirtschaftliche Verluste abzufangen. Für viele Branchen und Geschäftsbereiche waren die Maßnahmen auch ein Denkanstoß, der nachhaltig positive Zukunftsperspektiven für die Unternehmen und Mitarbeiter eröffnet. Dazu gehören unter anderem viele Maßnahmen im Zuge der fortschreitenden Digitalisierung.

Virtuelle Showrooms und Verkaufsplattformen. Der Siegeszug des Onlineshoppings beschäftigt Händler und Marken bereits seit Jahren. Die vorübergehenden Schließungen von Ladengeschäften zur Eindämmung der aktuellen Pandemie hat diesen Trend nicht nur unterstützt, sondern auch neue Konzepte hervorgebracht. Kleinere lokale Geschäfte haben sich vielerorts zusammengeschlossen und gemeinsame Onlineplattformen aufgebaut. Über diese können Konsumenten lokale Anbieter auch zukünftig als Alternative zu den großen Onlineplattformen nutzen.

Auch für Branchen, die traditionell stark auf ein Vor-OrtVerkaufserlebnis setzen, war die ungeplante Pause ein Denkanstoß. Die Automobilbranche beispielsweise setzt nach wie vor auf Showrooms und Autohändler vor Ort. Das Onlineangebot war bisher bei diesen und auch bei den Automobilherstellern selbst sehr unterschiedlich stark ausgeprägt ${ }^{1}$. Eine Testfahrt kann ein virtuelles Angebot auch in Zukunft nicht ersetzen. Ein großer Teil der Recherche für den Autokauf könnte sich jedoch dauerhaft in den Onlinebereich verlagern. Bereits heute sind Vergleichsplattformen, bei denen man Fahrzeugfeatures und Preise vergleichen kann, für viele Käufer der Startpunkt für den Autokauf. Anbieter, die darüber hinaus ein virtuelles Markenerlebnis bieten, bei dem die

1 https://www.consumerreports.org/buying-a-car/ coronavirus-pushing-car-dealerships-into-online-sales-andhome-delivery/

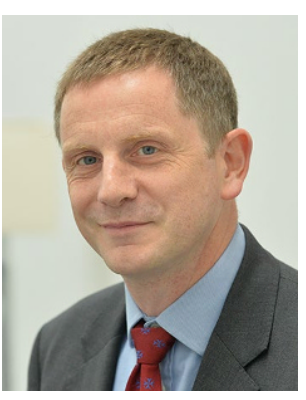

Liam Butler $(\bowtie)$ ist Vice President (VP) Sales von SumTotal EMEA, einem Anbieter für integrierte Lern-, Talent- und Workforce-Management-Lösungen. $\mathrm{Zu}$ seinen Aufgaben gehört es auch, dass er Marktveränderungen, Trends und die somit entstehenden Bedürfnisse von Unternehmen und Arbeitnehmern genau beobachtet. www.sumtotalsystems.de. Skillsoft@gcpr.net

SumTotal Systems GmbH, Berliner Allee 59, 40212 Düsseldorf, Deutschland

Käufer bereits im Vorfeld einen Eindruck vom Fahrerlebnis und der Ausstattung erhalten, realisieren einen klaren Verkaufsvorteil. Tesla, Hyundai oder Audi gehören dabei zu den Vorreitern, bei denen man online nicht nur sein Traummodell

Wirtschaftsinformatik \& Management 2020 • 12 (4): 274-276 https://doi.org/10.1365/s35764-020-00269-9

Online publiziert: 12 . Juni 2020

(c) Springer Fachmedien Wiesbaden $\mathrm{GmbH}$, ein Teil von Springer Nature 2020 
komplett konfigurieren kann ${ }^{2}$. In den virtuellen Showrooms erster Anbieter können potenzielle Käufer bereits von der heimischen Couch aus auf dem Fahrersitz oder auf der Rückbank Platz nehmen oder sich im Innenraum umsehen. Ein solches virtuelles Markenerlebnis erobert immer mehr Branchen von der digitalen Küchenplanung und der Möbelindustrie bis zu Sport- und Modemarken.

Kundendienst auf Distanz, aber trotzdem persönlich. Während bei vielen Firmen Verkaufspersonal aus Ladengeschäften und dem Direktvertrieb in den letzten Wochen in Kurzarbeit gehen musste, explodierten die Anfragen in Callund Contactcentern. Viele Branchen reagierten mit neuen Konzepten auf die Verlagerung der Kundenanfragen. Finanzund Versicherungsdienstleister beispielsweise bauten ihre virtuellen Beratungsangebote aus ${ }^{3}$. Statt Beratungsgespräche vor Ort mit Telefonberatung zu ergänzen, setzen zukunftsorientierte Dienstleister zum Beispiel auf Videokonferenzoptionen. Damit muss der Kunde nicht komplett auf den persönlicheren visuellen Kontakt zu seinem Berater verzichten. Dieses Konzept könnte sich für viele Nutzer und Berater auch in Zukunft durchsetzen, da es eine schnellere und flexiblere Terminfindung und Betreuung ermöglicht.

Ein weiterer innovativer Ansatz kommt aus der Automobilindustrie: Hyundai hatte bereits vor der Coronakrise seinen Online-Showroom mit persönlicher Beratung kombiniert. In einem Studio in Aschaffenburg stehen nicht nur vier Fahrzeugmodelle, sondern auch speziell geschulte Verkaufsberater bereit. Diese können Interessenten per Videochat um die Fahrzeuge führen und Fragen beantworten.

Im Zuge der allgemeinen Verlagerung in Richtung Onlineberatung gilt es für Unternehmen allerdings nicht nur, ihre Onlinekonzepte und verwendeten Technologien zu überdenken, sondern auch die personellen Ressourcen. Statt erfahrene Fachkräfte zu verlieren, können Weiterbildungsmaßnehmen diese bei Bedarf für einen Transit in die virtuelle Beratung qualifizieren.

Interaktion mit Partnern und Lieferanten. Im Bereich Partner- und Lieferanteninteraktion waren viele Unternehmen bisher noch wesentlich konservativer aufgestellt als im Kundenservice. Das übliche Vorgehen vieler Unternehmen

2 https://www.n-tv.de/auto/Autokauf-in-Zeiten-vonCorona-article21673278.html

3 https://unternehmen.handelsblatt.com/videoberatungversicherung.html baute auf einen großen Mitarbeiterstab, der vor Ort Verhandlungen und Schulungen durchführte. Besonders im Mittelstand zeigte sich mancher Lieferant oder Dienstleistungspartner virtuellen Kanälen gegenüber bisher als eher skeptisch. Laut Bitkom waren Ende 2019 nur 19 \% der Mittelständler softwareseitig für eine Digitalisierung aufgestellt ${ }^{4}$.

Die erzwungenen Kontaktbeschränkungen hatten diesbezüglich zumindest einen positiven Effekt: So mancher Skeptiker stellte fest, dass auch virtuelle Vertriebsprozesse, Treffen oder Schulungen besser funktionieren als gedacht. Der persönliche Kontakt bleibt sicher auch in Zukunft ein wichtiger Faktor. Die Zahl der Treffen und die vielen zurückgelegten Kilometer, die die „Road Warrior“ im Vertrieb fern von der Familie auf den Straßen zurücklegen, könnten sich jedoch deutlich reduzieren. Nicht nur in Hinsicht auf die Kostenvorteile sowie eine schnellere Umsetzung vieler Arbeitsprozesse eröffnet dies Vorteile.

Von Webinaren mit technischen Instruktionen für Installateure über das Onboarding von neuen Lieferanten über virtuelle Plattformen und mithilfe von Collaboration Tools sowie Videokonferenzen konnten viele Technologien in den letzten Wochen ihren praktischen Nutzen unter Beweis stellen.

Digitale Tools und Technologien. Digitale Technologien bilden eine wichtige Grundlage, um Arbeitsprozesse ohne persönlichen Kontakt umzusetzen. Ein wichtiger Schritt dabei ist die Integration von Geschäftssystemen und ihre Öffnung über Unternehmensgrenzen hinaus, um eine nahtlose Zusammenarbeit mit Partnern und Zulieferern zu ermöglichen. Webinare, Onlineschulungen, Collaboration-Plattformen und andere digitale Angebote lassen sich zudem für zahlreiche Geschäftsbereiche und Prozesse einsetzen. Die Nutzung von Videokonferenzlösungen ist in den letzten Wochen geradezu explodiert ${ }^{5}$. Die Möglichkeit, auch virtuell von Angesicht zu Angesicht zu kommunizieren, macht es möglich, trotz physischer Distanz eine persönliche Verbindung herzustellen. Das hilft beim Onboarding von Mitarbeitern, Partnern oder Lieferanten genauso wie beim Aufrechterhalten eines Gemeinschaftsgefühls, wenn Teams remote arbeiten. Mobiles und dezentrales Arbeiten ist generell ein Trend.

4 https://www.bitkom.org/sites/default/files/ 2019-10/191021_studie_digital-office-im-mittelstand.pdf

5 https://www.computerweekly.com/news/252481231/ Coronavirus-Soaring-collaboration-app-uptake-sees-homeworkers-clock-on-for-longer-hours 
Die Krisensituation zwang jedoch viele Unternehmen, ihre Ausstattung für digitale Systeme sowie Remote-Arbeitsplätze schneller als geplant aufzustocken. Laut einer Umfrage des Digitalverbands Bitkom arbeitet zurzeit jeder Zweite (49 \%) ganz oder zumindest teilweise im Homeoffice und kann sich vorstellen, auch in Zukunft teilweise remote $\mathrm{zu}$ arbeiten ${ }^{6}$.

Keine Transformation ohne den Schlüsselfaktor Mitarbeiter. Zwar bildet die Implementierung digitaler Technologie eine wichtige Grundlage für die Umsetzung einer Transformation. Genauso wichtig ist es jedoch, die benötigten Fähigkeiten und Qualifikationen bei den Mitarbeitern aufzubauen. Eine Studie von Skillsoft zeigte, dass im ersten Quartal 2020 die Mehrzahl der befragten Unternehmen in Deutschland, Frankreich und Großbritannien fehlende Qualifikationen für die digitale Transformation als eine der größten Herausforderungen sieht ${ }^{7}$. Nicht nur der Umgang mit neuen Technologien und Tools ist ein Bereich, in dem Mitarbeiter immer häufiger geschult werden müssen. Ein flexibel und schnell anwendbares System zur Mitarbeiterschulung gehört für fast alle Arbeitsbereiche zu den Schlüsselfaktoren, um auf neue Situationen und Anforderungen reagieren zu können. Das betrifft Mitarbeiter, die beispielsweise Hygienevorschriften beim Kundenkontakt einhalten, mit einem saisonalen Ansturm in Geschäften oder den aktuellen Beschränkungen und Abstandsregeln umgehen müssen genauso wie solche, bei denen sich der Kontakt mit Kunden, Kollegen oder Partnern immer stärker auf virtuelle Kanäle verlagert.

Mithilfe digitaler HR-, HCM- und Lernplattformen werden Mitarbeiterschulungen heute wesentlich flexibler und können bei Bedarf jederzeit und von jedem Ort aus umgesetzt werden. Die Systeme helfen auch dabei, zu erkennen, bei welchen Mitarbeitern sich eine Weiterbildung oder Umschulung anbieten würde, falls durch Umstellungen im Unternehmen - beispielsweise eine Verlagerung der Beratung in Onlinekanäle - einige Aufgaben bzw. Rollen wegfallen und an anderer Stelle neue Jobs entstehen.

Fazit. Unser Arbeitsalltag unterliegt immer schnelleren Veränderungen. Neue Technologien sowie insgesamt schnell wechselnde Anforderungen von Markt und Kunden beeinflussen auch die Anforderungen an die Mitarbeiter. Wer in der Vergangenheit dachte, mit einer soliden Ausbildung für

6 https://www.bitkom.org/Presse/Presseinformation/ Corona-Pandemie-Arbeit-im-Homeoffice-nimmt-deutlich-zu 7 https://bit.ly/3bpBIbv sein Arbeitsleben gewappnet zu sein, der sieht sich heute einem lebenslangen Lernprozess gegenüber, um für die ständig wechselnden Anforderungen und Rollen im Berufsalltag gerüstet zu sein. Fähige Mitarbeiter bleiben auch in Zukunft das Rückgrat erfolgreicher Unternehmen, daher lohnt es sich, in die Qualifikation von Mitarbeitern zu investieren. 